\title{
Engineering of a Dynamic Multi-Frequency Antenna
}

\author{
Clive Sugama \\ College of Engineering \\ Colorado State University \\ Fort Collins, CO 80523
}

\begin{abstract}
Engineering a dynamic multi-frequency antenna provides the advantage of reducing overall antenna footprints and life cycle costs for supporting multiple Radio Frequency (RF) systems. Maintenance, logistics support and procurement costs associated with various antenna system manufacturers' increase overall RF system life cycle costs. At the present time, multiple RF antennas developed by different manufacturers provide capabilities to shipboard platforms. Communication on shipboard platforms is necessary for line of sight and satellite communication operations.
\end{abstract}

Antenna design software called Antenna Magus was used to analyze the capabilities of multi-frequency capable antennas. Parameters such as focal depth, dish diameter, dish to feed spacing, waveguide width, waveguide height, waveguide length, aperture width, aperture height, and flare length were automatically adjusted to support frequencies from $1 \mathrm{GHz}$ to 300 GHz. A conceptual method for adjusting the dish diameter and dish to feed spacing was proposed to assist with providing a dynamic approach to a multi-frequency antenna.

Additional design criteria would have to be considered to adhere to standards and manage risks during the early design stages of the system life cycle. Interfacing with legacy RF systems would be a technical challenge for interoperability. This paper presents engineering requirements and approach for reducing the amount of antennas manufactured by proprietary vendors by implementing an adjustable antenna to support SATCOM, and EHF capabilities.

Keywords- Radio Frequency; Communication Systems; MultiFrequency; Antenna; Engineering

\section{INTRODUCTION}

Since 1983, the number of antennas aboard surface ships has roughly doubled [1]. As technology advances, the warfighter is in need of various RF capabilities to stay ahead of adversaries. RF communications are critical to transmit and receive information for military operations. Space limitations constrain the amount of RF antennas on shipboard platforms.

Extensive engineering analysis had been performed and various prototypes for consolidated antennas were developed over the past 15 years. One of the first prototypes was the Advanced Multifunction RF (AMRF) Concept [2] and most recently was the EW/IO/Comm Advanced Development Model (ADM) [3]. Furthermore, extensive systems engineering analysis from HF to SHF has been performed on shipboard RF systems as well as proposed architectures for the development of a new prototype, LowRIDR [4].

Multiple manufacturers provide antennas with similar and unique capabilities. The procurement of these antenna systems are manufacturer dependent, and are within different stages of the system life cycle. As systems become obsolete, replacements and parts for these antennas become scarce. Obtaining various antennas from manufacturers to provide certain capabilities incurs a high cost along with specific vendor support.

RF capabilities are separated amongst various antennas and an adjustable multi-frequency antenna to support a wide range of frequencies such as SHF and EHF would be beneficial. The need for consolidating RF antennas would reduce the number of system owners to a select few as well as the amount of space required. Maintaining multiple RF antennas involves various subject matter experts, stakeholders and personnel associated with the system's program of record. Collaborating amongst different stakeholders to troubleshoot RF system issues is common when maintaining continuity of RF communication capabilities. Coordinating with system owners to assist with troubleshooting efforts, testing, and managing risks is an ongoing effort.

The dynamic multi-frequency RF system would reduce the amount of manufacturer specific antennas. This system would include an adjustable dish, Communication Group, and assemblies that support SHF / EHF capabilities. The adjustable dish would be dynamic in nature with the ability to contract, expand, radiate, and receive. The feed horn assemblies would support SHF and EHF frequencies that span from $1 \mathrm{GHz}$ to 300 GHz. Satellite, voice, and data link Line of Sight (LOS) / Beyond LOS (BLOS) would be supported by the assembly / assemblies. The communication group would involve modern / legacy systems that would encrypt / decrypt and modulate / demodulate various forms of communication such as internet, messaging, and voice over Internet Protocol (IP). Figure 1 illustrates the architecture of the dynamic multi-frequency RF system that operates at various frequency ranges. 


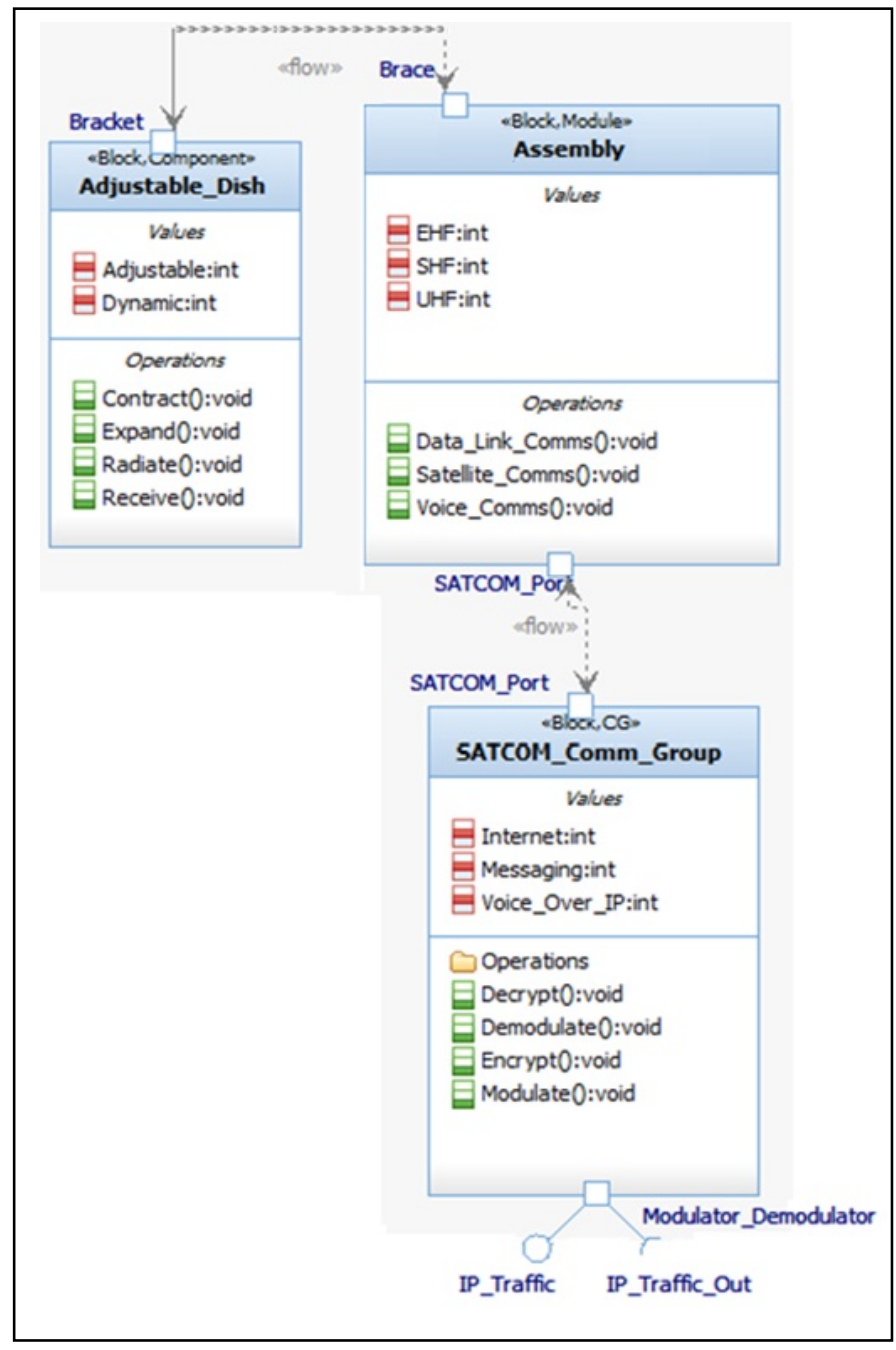

Figure 1: Dynamic Multi-Frequency

\section{ANTENNA ANALYSIS}

Antenna Magus is a software application used to simulate antenna performance to aid RF antenna design. This software assists with determining physical properties and radiation patterns to suggest the selection of particular types of antennas to meet specific needs. For multi-frequency capable antennas, comparisons were made to identify potential options for replacing currently fielded antennas with intent to consolidate capabilities. Antenna Magus has the capability to filter various types of antennas based on frequency, size, capability, and other performance parameters. Estimating the performance within this software allowed settings to be adjusted automatically for stable use. Antenna magus also depicted appropriate use of antennas for directional operational purposes.
Parabolic reflector antennas were considered for SATCOM and EHF communications use. The high level of gain that can be achieved by using a parabolic reflector is one of the main reasons they are used [5]. Utilizing Antenna Magus assisted with comparing various parabolic antennas. SATCOM antennas that were capable of supporting frequencies from $1 \mathrm{GHz}$ to $300 \mathrm{GHz}$ were analyzed which are included in Table 1.

Table 1: $1 \mathrm{GHz}$ to $300 \mathrm{GHz}$ Capable Parabolic Antennas

\begin{tabular}{|c|c|}
\hline 1 & Horn-fed Offset Cassegrain Reflector Antenna \\
\hline 2 & Pattern-fed Cassegrain Reflector Antenna \\
\hline 3 & Horn-fed Parabolic Reflector Antenna \\
\hline 4 & Parabolic Reflector theoretical pattern fed \\
\hline 5 & Horn-fed Truncated Parabolic Reflector Antenna \\
\hline 6 & Horn-fed Cassegrain Reflector Antenna \\
\hline 7 & Offset pattern-fed Cassegrain Reflector Antenna \\
\hline 8 & Horn-fed Gregorian Dual-Reflector Antenna \\
\hline 9 & Pattern-fed Gregorian Dual-Reflector Antenna \\
\hline 10 & Horn-fed Offset Gregorian Reflector Antenna \\
\hline 11 & Offset Pattern-fed Gregorian Reflector \\
\hline 12 & Offset symmetric horn-fed Parabolic Reflector \\
\hline 13 & Offset symmetric pattern-fed Parabolic reflector \\
\hline
\end{tabular}

These antennas are capable of supporting frequencies $1 \mathrm{GHz}$ - 40GHz (SATCOM), and 30GHz - 300GHz (EHF). SATCOM and EHF frequencies would be used to support required capabilities using components supporting them respectively. Various properties of the Horn-fed Parabolic Reflector Antenna was analyzed to include Focal depth (F), Dish Diameter (D), Dish to feed spacing (S), Waveguide width (Wg), Waveguide height (Hg), Waveguide length (Lg), Aperture width (Wa), Aperture height (Ha), and Flare length (Lf). These properties revealed that physical adjustments would have to be made on the feed horn aperture and the dish to produce required performance values. Different views of the horn, reflector, and the dish are shown in Figure 2. 


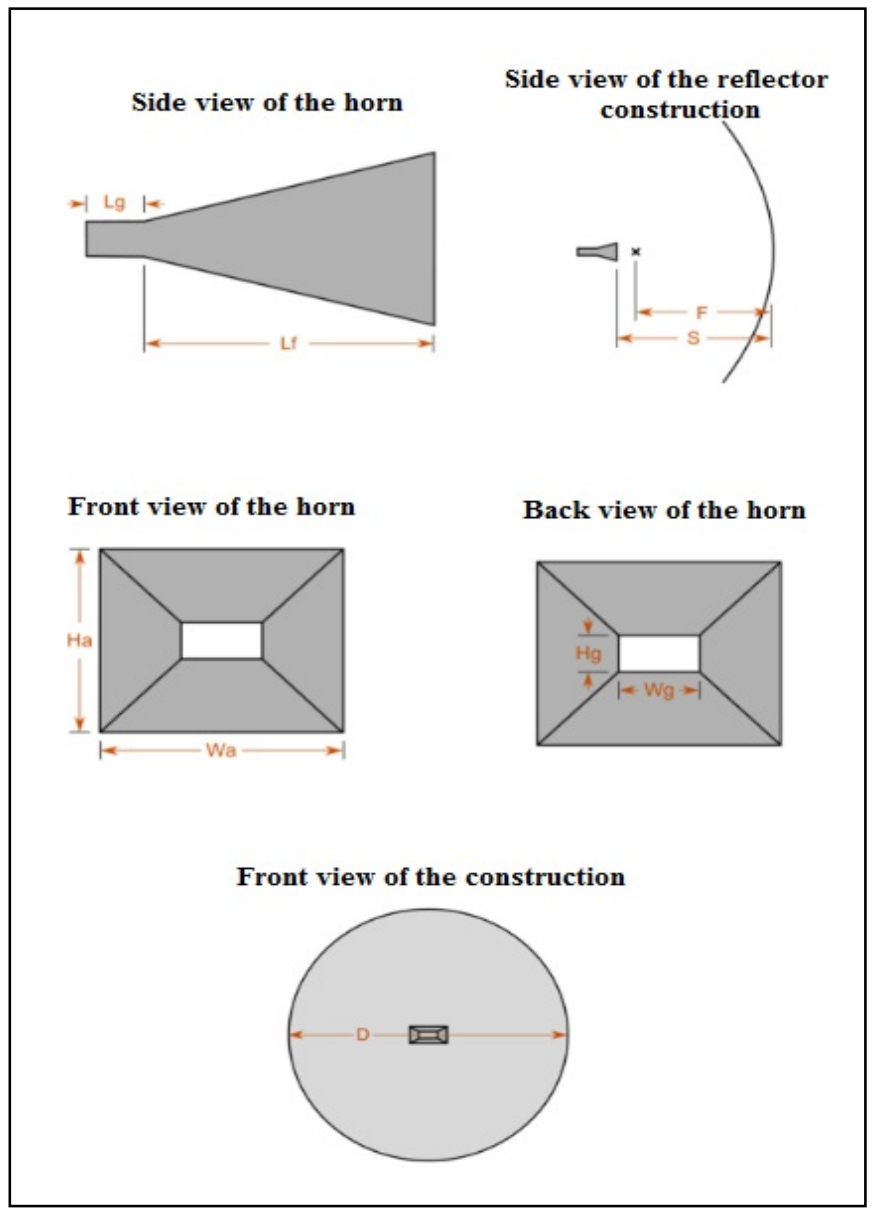

Figure 2: Antenna Magus Design Views of a Horn-fed Parabolic Reflector Antenna [6]

The SATCOM and EHF frequencies were set within the Antenna Magus software to observe results of the simulation. The physical parameters were automatically adjusted by the Antenna Magus software to meet the performance requirements. As the frequency increased, the physical parameters of the antenna were reduced. This assessment indicates the need for a dynamic antenna that can adjust its physical attributes in order to meet mission requirements as shown in Table 2.

Table 2: Antenna Magus Simulation Results for SATCOM and EHF for a Horn-fed Parabolic Reflector Antenna

\begin{tabular}{|c|c|c|c|}
\hline & & SATCOM & EHF \\
\hline \multicolumn{2}{|c|}{ Frequency Range } & $1 \mathrm{GHz}-40 \mathrm{GHz}$ & $30 \mathrm{GHz}-300 \mathrm{GHz}$ \\
\hline \multicolumn{2}{|c|}{ Center Frequency } & $20.5 \mathrm{GHz}$ & $165 \mathrm{GHz}$ \\
\hline Name & Description & Value & Value \\
\hline $\mathrm{F}$ & Focal depth & $379.4 \mathrm{~mm}$ & $47.14 \mathrm{~mm}$ \\
\hline $\mathrm{D}$ & Dish diameter & $552.4 \mathrm{~mm}$ & $68.63 \mathrm{~mm}$ \\
\hline $\mathrm{S}$ & Dish to feed spacing & $379.4 \mathrm{~mm}$ & $47.14 \mathrm{~mm}$ \\
\hline $\mathrm{Wg}$ & Waveguide width & $11.48 \mathrm{~mm}$ & $1.426 \mathrm{~mm}$ \\
\hline $\mathrm{Hg}$ & Waveguide height & $5.740 \mathrm{~mm}$ & $713.1 \mu \mathrm{m}$ \\
\hline $\mathrm{Lg}$ & Waveguide length & $14.62 \mathrm{~mm}$ & $1.817 \mathrm{~mm}$ \\
\hline $\mathrm{Wa}$ & Aperture width & $21.95 \mathrm{~mm}$ & $2.727 \mathrm{~mm}$ \\
\hline $\mathrm{Ha}$ & Aperture height & $16.83 \mathrm{~mm}$ & $2.091 \mathrm{~mm}$ \\
\hline Lf & Flare length & $38.78 \mathrm{~mm}$ & $4.818 \mathrm{~mm}$ \\
\hline
\end{tabular}

The Horn-fed Parabolic Reflector antenna was analyzed to simulate the radiation pattern. Estimating the performance for the Horn-fed Parabolic Reflector Antenna produced a radiation pattern within the Antenna Magus Software. This antenna is capable of SATCOM and EHF frequencies ranging from 1 $\mathrm{GHz}$ to $300 \mathrm{GHz}$. Each performance simulation was completed to observe parameters relative to each set of frequency ranges (SATCOM and EHF). The focal depth, dish diameter, and dish to feed spacing were analyzed to meet the optimal conditions to meet the radiation pattern. A radiation pattern was observed when estimating the performance through the software. In this antenna, the parabolic reflector is fed by a pyramidal horn antenna, which in turn is fed using a waveguide port. In practice, the horn may be fed using a waveguide system, or alternatively, using a coaxial probe feed (waveguide-to-coaxial transition) [6]. As shown in Figure 3, the radiation pattern is depicted for SATCOM and EHF after performing the simulation.

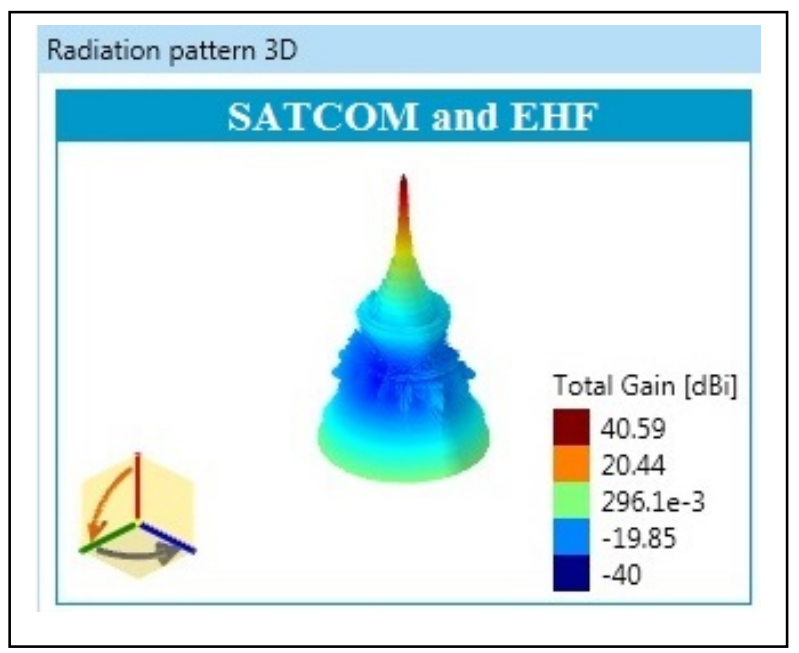

Figure 3: Antenna Magus Radiation Pattern of SATCOM and EHF Capabilities

A dynamic antenna would be required to adjust focal depth, dish diameter, and dish to feed spacing. Slide rail actuators have the ability to reduce and increase length of this antenna. These actuators would adjust the size of the dish to the corresponding band of frequencies required. Another set of actuators would adjust the distance between the feed horn(s) to the dish to complement the capability required. Software on the communications group would control these parameters to complement the frequencies desired. Figure 4 depicts the dynamic concept where the dish size expands and contracts as well as the self-adjusting feed horn. Using an adjustable antenna dish can allow the operator to customize their antenna system setup based on mission requirements.

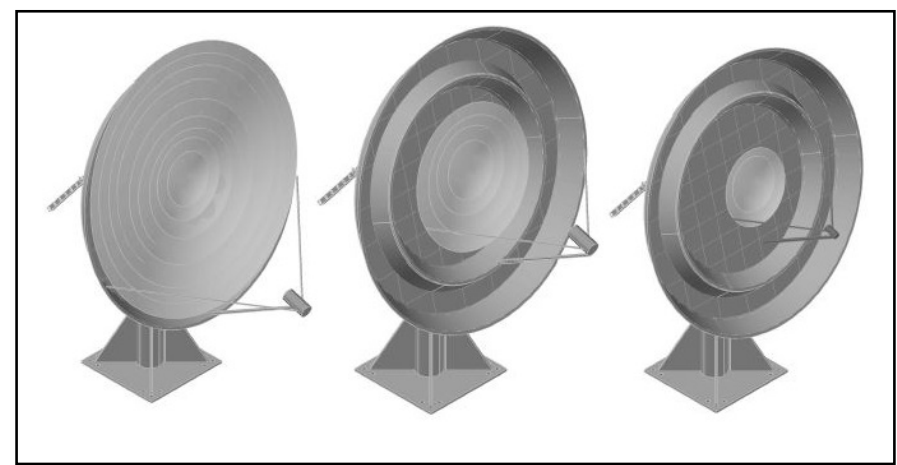

Figure 4: Conceptual Adjustable Dish Depiction; Adapted from [7] 
The feed horn would have to be adjustable as well to support frequencies of $1 \mathrm{GHz}$ to $300 \mathrm{GHz}$. The feed horn's waveguide width, waveguide height, waveguide length, aperture width, aperture height, and flare length would require flexible adjustments to meet performance parameters. The use of multiple feed horns is another alternative that would provide similar capabilities but with a limited range of supportable frequencies.

\section{DESIGN CONSIDERATIONS}

Standards would be followed to assist with designing the dynamic multi-frequency antenna. Standards categories include RF transfer, information assurance, RF testing, emissions, safety, and disturbance. The goals for each of the standards have been identified in order to facilitate requirement development. This standards profile is shown in Table 4.

Table 4: RF Antenna Standards Profile

\begin{tabular}{|c|c|}
\hline Standards Categories & Typical Standards \\
\hline $\begin{array}{l}\text { RF Transfer: } \\
\text { - Frequency Range and Rate } \\
\text { - Noise limits } \\
\text { - RF Exposure } \\
\text { - EMI Control }\end{array}$ & $\begin{array}{l}\text { Goal: Operate within RF rules and } \\
\text { protocol } \\
\text { - Broadcast standards } \\
\text { - Signal to noise ratio standards } \\
\text { - FCC Regulations, IEEE C95.1 } \\
\text { - MIL-STD-461G, IEEE C63.12- } \\
\text { 2015, IEEE C63.2-2009, IEEE } \\
\text { C37.90.2-2004 }\end{array}$ \\
\hline $\begin{array}{l}\text { Information Assurance: } \\
\text { - RF COMSEC } \\
\text { - Network Security } \\
\text { - Physical Security } \\
\text { - Software Security } \\
\end{array}$ & $\begin{array}{l}\text { Goal: Capable of passing an IA } \\
\text { audit } \\
\text { - PKI, EKMS standards and } \\
\text { protocol } \\
\text { - DISA Information Assurance } \\
\text { Support Environment Reqs. } \\
\text { - TEMPEST physical security } \\
\text { standards } \\
\text { - ISO/IEC } 27034\end{array}$ \\
\hline $\begin{array}{l}\text { RF Testing: } \\
\text { - Standard Test Procedures for } \\
\text { Antennas } \\
\text { - Sites performing Emission } \\
\text { testing } \\
\text { - Compliance of Transmitters } \\
\text { - Electrostatic Discharge } \\
\text { - Environmental } \\
\text { - RF Absorption }\end{array}$ & $\begin{array}{l}\text { Goal: Perform and meet outlined } \\
\text { test procedures } \\
\text { - IEEE 149-1977 } \\
\text { - IEEE C63.7-2015, IEEE C63.7- } \\
\text { 2005 } \\
\text { - IEEE C63.36-2015 } \\
\text { - IEEE C63.16-2016 } \\
\text { - IEEE 1613.1-2013 } \\
\text { - IEEE 1128-1998 }\end{array}$ \\
\hline $\begin{array}{l}\text { Emissisons: } \\
\text { - FM and TV Broadcast } \\
\text { Receivers } \\
\text { - Land-Mobile Transmitters } \\
\text { - Low Voltage Electrical } \\
\text { Equipment }\end{array}$ & $\begin{array}{l}\text { Goal: Stay within acceptable RF } \\
\text { emissions boundaries } \\
\text { - IEEE 187-2003 } \\
\text { - IEEE 377-1980 } \\
\text { - IEEE C63.4-2014 }\end{array}$ \\
\hline $\begin{array}{l}\text { Safety: } \\
\text { - RF Safety Program 3kHz to } \\
\text { 300GHz } \\
\text { - Human Exposure to EMI }\end{array}$ & $\begin{array}{l}\text { Goal: Adhere to RF safety } \\
\text { precautions } \\
\text { - IEEE C95.7-2014 } \\
\text { - IEEE C95.7-2014, IEEE C95.1- } \\
\text { 2005, IEEE C95.3.1-2010 }\end{array}$ \\
\hline $\begin{array}{l}\text { - Radio and IT Equipment } \\
\text { - Radio Noise }\end{array}$ & $\begin{array}{l}\text { Goal: Meet acceptable signal to } \\
\text { noise ratio requirements } \\
\text { - IEEE C63.022-1996, IEEE } \\
\text { C63.011-2000 } \\
\text { - IEEE C63.5-2006, IEEE C63.4- } \\
\text { 2003 }\end{array}$ \\
\hline
\end{tabular}

Determining RF functions that operate concurrently with each other without interference would be ideal for multifunctional operational scenarios. Interference problems between adjacent antennas increase the likelihood of dropped communication links. Simulated RF interference testing would be conducted during transmitting / receiving scenarios to identify any RF signal degradation. While the antenna is transmitting and receiving on a particular frequency, various frequency ranges would be used for interference testing to identify any issues. Constraints would be identified with respect to having multiple $\mathrm{RF}$ transmissions being radiated simultaneously.

\section{TECHNICAL CHALLENGES}

Technical challenges would involve different areas of constraints to include size, weight, and power limitations. Interfacing with legacy systems would also have to be considered to perform operations with shipboard platforms. Electromagnetic Interference (EMI) would be a technical challenge that would have to be determined in order to allow functionality amongst neighboring communications systems.

Interfacing with current and legacy systems connected to shipboard antennas may inhibit challenges outside of simple interface adapters. Physical interfaces should be considered to connect to legacy systems. Interoperability between current and legacy systems is necessary to incorporate a dynamic multi-frequency antenna within an existing communication system domain. Physical and functional integration testing is also necessary in order to verify full interoperability. Access to interfacing systems may be a challenge due to not being able to control the scheduling of resource availability for collaboration between legacy RF system owners.

The placement of these antennas on a shipboard platform would need to be considered to identify blockage zones along with platform strength. Modal testing of the antenna mounting area of the shipboard platform to obtain strength values would establish a baseline design. Failure to acknowledge the strength of the shipboard platform would pose a risk resulting in engineering an antenna that exceeds the limitations of the platform strength. Adapter plates would also be considered for shipboard platforms that need to be retrofitted due to constrained mounting locations.

Shipboard antennas encounter harsh conditions that would potentially affect operation ranging from the surrounding area to non-visible EMI. Environmental conditions such as extreme hot / cold temperatures, moisture, wind speed, sun exposure, wave vibration, and salt water would affect the newly designed antenna if not properly considered. Anti-corrosive and durable components would be incorporated to ensure longevity of the system. The designed antenna would go through vibration, environmental, and EMI testing. Mounting a newly designed antenna to a large motion table for testing, would simulate the pitch and roll movement of a shipboard platform.

\section{CONCLUSION}

Developing the dynamic multi-frequency RF antenna would reduce the amount of antennas required by different manufacturers on shipboard platforms. The dynamic multifrequency RF antenna architecture includes an adjustable dish with assemblies connecting to a communications group to transmit and receive information. Design considerations such as standards would be adhered to for RF transfer, information 
assurance, RF testing, emissions, safety, and disturbance. Technical challenges with EMI and interfacing current / legacy systems would require ongoing risk management practices.

Utilizing the antenna design software Antenna Magus identified various parabolic antennas capable of supporting frequencies $1 \mathrm{GHz}$ to $300 \mathrm{GHz}$. Adjusting the dish size and feed horn aperture allowed for SATCOM and EHF frequencies to be supported. The dish diameter and feed to dish configuration would be capable of contracting / expanding. A feed horn with similar features of contracting / expanding to provide multi-frequency capabilities or the use of multiple feed horns to support frequencies within the $1 \mathrm{GHz}$ to $300 \mathrm{GHz}$ range would provide a single antenna solution from a single manufacturer.

\section{REFERENCES}

[1] G. Winters, “A Short History of NSWC Dahlgren's Involvement in the Shipboard Electromagnetic Compatibility Improvement Program,” Leading Edge, vol. 7, pp.29.

[2] G. C. Tavik, C. L. Hilterbrick, J. B. Evins, J. J. Alter, J. G. Crnkovich, J. W. de Graaf, W. Habicht II, G. P. Hrin, S. A. Lessing, D. C. Wu, and S. M. Hagewood, "The Advanced Multifunction RF Concept,” IEEE Trans. On Microwave Theory and Techniques, Vol. 53, No. 3. March 2005.

[3] J. A. Molnar, I. Corretjer, and G. Tavik, "Integrated Topside Integration of Narrowband and Wideband Array Antennas for Shipboard Communications," Millitary Communication Connference-Track 5-Communications and Networks, 2011.

[4] A. Leese de Escobar, K. Beauvais, D. Brandon, J. Brewster, S. Chieh, M.I. Civerolo, E. Constantino, S. Loui, O. Nayfeh, J. Neff, J. Reddan, E. Yu, "Low-band RF Intelligent Distribution Resource (LowRIDR),” SPAWAR Systems Center Pacific TR 2059, March 2014.

[5] "Parabolic Reflector Antenna Gain | Formula Calculation Equation | Tutorial", Radio-electronics.com, 2017. [Online]. Available: http://www.radioelectronics.com/info/antennas/parabolic/parabolic-reflectorantenna-gain.php.

[6] "ANTENNA MAGUS | The leading Antenna Design Software tool. | Antenna Design. Simplified", Antennamagus.com, 2017. [Online]. Available: http://www.antennamagus.com/.

[7] V. Michl, "SatAntenna," CAD Forum - Block: Joist Hanger (Ceiling), 28-Sep-2009. [Online]. Available: http://www.cadforum.cz/catalog_en/block.asp?blk=5111. [Accessed: 03-Jul-2018]. 